\title{
Kinetics of hydrogenation of aqueous levulinic acid over bimetallic $\mathrm{Ru}-\mathrm{Ni} / \mathrm{MMT}$ catalyst
}

Gayatri B. Kasar, ${ }^{[a, b]}$ Rucha S. Medhekar, ${ }^{[a]}$ P.N. Bhosale, ${ }^{[b]}$ Chandrashekhar V. Rode ${ }^{*[a]}$

[a] Chemical Engineering and Process Development Division, CSIR-NCL, Dr. Homi

Bhabha Road, Pashan, Pune-411008, India

[b] Materials Research Laboratory, Department of Chemistry, Shivaji University,

Kolhapur-416004, India.

\# Supporting Information 


\section{Table of contents:}

Table S1: Catalyst screening.

Table S2: Kinetic Models

Table S3: The values of rate constants estimated from model II (the second model considered) at different temperatures

Table S4: B/L ratios of catalysts

Table S5: Thermodynamic properties of the reaction.

Figure S1: Py-IR of catalysts.

Figure S2: Concentration and selectivity Vs time profile of $0.5 \% \mathrm{Ru}-5 \% \mathrm{Ni} / \mathrm{MMT}$ catalyst.

Figure S3: Effect of $0.5 \% \mathrm{Ru}-5 \% \mathrm{Ni} / \mathrm{MMT}$ catalyst amount.

Figure S4: Graph of rate of reaction obtained from model-I (the first model considered) Vs rate of reaction obtained from experimental at temp $160^{\circ} \mathrm{C}$.

Figure S5: Graph of rate of reaction obtained from model-I (the first model considered) Vs rate of reaction obtained from experimental at temp $235^{\circ} \mathrm{C}$

Figure S6: Arrhenius plot of rate constant $\mathrm{k}_{\mathrm{LA}}$ Vs 1/T from Model I(the first model considered)

Figure S7: Arrhenius plot of rate constant $\mathrm{k}_{\mathrm{H} 2}$ Vs 1/T from Model I(the first model considered)

Figure S8: Arrhenius Plot for $\mathrm{k}_{3}$ obtained from Model II (the second model considered)

Figure S9: Arrhenius Plot for $\mathrm{K}_{\mathrm{LA}}$ obtained from Model II (the second model considered)

Figure S10: Arrhenius Plot for $\mathrm{K}_{\mathrm{H} 2}$ obtained from Model II (the second model considered)

Figure S11: Detailed derivation of the LHHW models used 
Figure S12: Report of POLYMATH 6.1 for model I(the first model considered)

Figure S13: Report of POLYMATH 6.1 for model II (the second model considered)

1] Table S1: Catalyst screening.

\begin{tabular}{llllll}
\hline Sr. & Catalyst & Conv, $\%$ & \multicolumn{3}{l}{ Selectivity, $\%$} \\
\cline { 4 - 5 } No & & & GVL & PA & $1,4-\mathrm{PeDO}$ \\
\hline 1 & MMT & 0 & 0 & 0 & 0 \\
2 & $0.5 \mathrm{wt} \% \mathrm{Ru} / \mathrm{MMT}$ & 81 & 95 & 4 & 1 \\
3 & $5 \mathrm{wt} \% \mathrm{Ni} / \mathrm{MMT}$ & 80 & 91 & 7 & 2 \\
4 & $0.5 \mathrm{wt} \% \mathrm{Ru}-1 \mathrm{wt} \% \mathrm{Ni} / \mathrm{MMT}$ & 86 & 96 & 2 & 2 \\
5 & $0.5 \mathrm{wt} \% \mathrm{Ru}-3 \mathrm{wt} \% \mathrm{Ni} / \mathrm{MMT}$ & 89 & 98 & 2 & 0 \\
6 & $0.5 \mathrm{wt} \% \mathrm{Ru}-5 \mathrm{wt} \% \mathrm{Ni} / \mathrm{MMT}$ & 91 & 100 & 0 & 0 \\
7 & $1 \mathrm{wt} \% \mathrm{Ru}-5 \mathrm{wt} \% \mathrm{Ni} / \mathrm{MMT}$ & 94 & 90 & 8 & 2 \\
8 & $1.5 \mathrm{wt} \% \mathrm{Ru}-5 \mathrm{wt} \% \mathrm{Ni} / \mathrm{MMT}$ & 100 & 90 & 8 & 2 \\
9 & $2 \mathrm{wt} \% \mathrm{Ru}-5 \mathrm{wt} \% \mathrm{Ni} / \mathrm{MMT}$ & 100 & 90 & 8 & 2 \\
10 & $3 \mathrm{wt} \% \mathrm{Ru}-5 \mathrm{wt} \% \mathrm{Ni} / \mathrm{MMT}$ & 100 & 90 & 7 & 3 \\
\hline
\end{tabular}

Reaction conditions: Levulinic Acid, 4g; Solvent, $95 \mathrm{~mL}$; catalyst, $0.25 \mathrm{~g} ; \mathrm{Temp}, 220^{\circ} \mathrm{C} ; \mathrm{H}_{2}$ pressure, 250psi; agitation speed, 1000rpm, Reaction time; $5 \mathrm{~h}$. 
2] Table S2: Kinetic Models

\begin{tabular}{|c|c|c|c|}
\hline Model & Mechanism & Initial rate expression & $\mathrm{R}^{2}$ \\
\hline I & $\begin{array}{l}\text { Dissociative adsorption } \\
\text { of } \mathrm{H}_{2} \text { and chemical } \\
\text { reaction as rate limiting } \\
\text { step }\end{array}$ & $-\mathbf{r}_{3}=\frac{\mathbf{k}_{3} \mathbf{K}_{\mathrm{LA}} \mathbf{L} \mathbf{A}_{0} \mathbf{K}_{\mathrm{H}_{2}} \mathbf{H}_{\mathbf{2}_{0}}}{\left(1+\mathbf{K}_{\mathrm{LA}} \mathbf{L} \mathbf{A}_{0}+\sqrt{\mathbf{K}_{\mathrm{H}_{2}} \mathbf{H}_{\mathbf{2}_{0}}}\right)^{3}}$ & 0.9922382 \\
\hline II & $\begin{array}{l}\text { Non-dissociative } \\
\text { adsorption of } \mathrm{H}_{2} \text { and } \\
\text { chemical reaction as } \\
\text { rate limiting step }\end{array}$ & $-\mathbf{r}_{3}=\frac{\mathbf{k}_{3} \mathbf{K}_{\mathrm{LA}} \mathbf{L} \mathbf{A}_{0} \mathbf{K}_{\mathrm{H}_{2}} \mathbf{H}_{\mathbf{2}_{0}}}{\left(1+\mathbf{K}_{\mathrm{LA}} \mathbf{L} \mathbf{A}_{0}+\mathbf{K}_{\mathrm{H}_{2}} \mathbf{H}_{\mathbf{2}_{0}}\right)^{2}}$ & 0.97104 \\
\hline III & $\begin{array}{l}\text { Dissociative adsorption } \\
\text { of } \mathrm{H}_{2} \text { and adsorption of } \\
\text { LA as rate limiting step }\end{array}$ & $-\mathbf{r}_{1}=\frac{\mathbf{k}_{\mathbf{1}} \mathbf{L} \mathbf{A}_{\mathbf{0}}}{\left(1+\sqrt{\mathbf{K}_{\mathbf{H}_{2}} \mathbf{H}_{\mathbf{2}_{0}}}\right)}$ & 0.651418 \\
\hline IV & $\begin{array}{l}\text { Dissociative adsorption } \\
\text { of } \mathrm{H}_{2} \text { and adsorption of } \\
\mathrm{H}_{2} \text { as rate limiting step }\end{array}$ & $-\mathbf{r}_{2}=\frac{\mathbf{k}_{2} \mathbf{H}_{2_{0}}}{\left(1+\mathbf{K}_{\mathrm{LA}} \mathbf{L} \mathbf{A}_{0}\right)^{2}}$ & 0.871860 \\
\hline V & $\begin{array}{l}\text { Non-dissociative } \\
\text { adsorption of } \mathrm{H}_{2} \text { and } \\
\text { adsorption of LA as rate } \\
\text { limiting step }\end{array}$ & $-\mathbf{r}_{1}=\frac{\mathbf{k}_{1} \mathbf{L} \mathbf{A}_{0}}{\left(1+\mathbf{K}_{\mathbf{H}_{2}} \mathbf{H}_{\mathbf{2}_{0}}\right)}$ & 0.98363 \\
\hline VI & $\begin{array}{l}\text { Non-dissociative } \\
\text { adsorption of } \mathrm{H}_{2} \text { and } \\
\text { adsorption of } \mathrm{H}_{2} \text { as rate } \\
\text { limiting step }\end{array}$ & $-\mathbf{r}_{2}=\frac{\mathbf{k}_{2} \mathbf{H}_{\mathbf{2}_{0}}}{\left(1+\mathbf{K}_{\mathrm{LA}} \mathbf{L} \mathbf{A}_{0}\right)}$ & 0.87186 \\
\hline
\end{tabular}


3] Table S3: The values of rate constants estimated from model II (the second model considered) at different temperatures:

\begin{tabular}{|c|c|c|c|}
\hline $\begin{array}{c}\text { Temperature } \\
\left({ }^{\circ} \mathrm{C}\right)\end{array}$ & $\mathrm{k}_{3}$ & $\mathrm{~K}_{\mathrm{LA}}$ & $\mathrm{K}_{\mathrm{H} 2}$ \\
\hline 160 & 3.882916 & 1.014883 & 0.697095 \\
\hline 220 & 101.9993 & 0.038483 & 0.690822 \\
\hline 235 & 101.9992 & 0.040086 & 0.756886 \\
\hline
\end{tabular}


4] Table S4: B/L ratios of catalysts

\begin{tabular}{|c|c|c|}
\hline \multirow{2}{*}{ Sr.No. } & Catalyst & B/L ratio \\
& & \\
\hline 1 & & \\
\hline 2 & Bare MMT-K-10 & 7.96 \\
\hline 3 & $0.5 \% \mathrm{Ru} / \mathrm{MMT}$ & 3.52 \\
\hline 4 & $5 \% \mathrm{Ni} / \mathrm{MMT}$ & 2.31 \\
\hline 5 & $0.5 \% \mathrm{Ru}+5 \% \mathrm{Ni} / \mathrm{MMT}$ & 0.05 \\
\hline
\end{tabular}


5] Table S5: Thermodynamic properties of the reaction.

\begin{tabular}{|c|c|}
\hline \multicolumn{2}{|c|}{ For Surface Reaction } \\
\hline Equation of $\mathrm{k}_{3}$ & $\mathrm{k}_{3}=3.83 * 10^{6 *} \mathrm{e}^{(-44098.287 / \mathrm{R} * \mathrm{~T})}$ \\
\hline Activation energy $\mathrm{Ea}(\mathrm{kJ} / \mathrm{mol})$ & 44.098 \\
\hline $\mathrm{A}_{03}\left(\mathrm{gcat}^{2} / \mathrm{mol}^{2} \mathrm{hr}\right)$ & $3.83 * 10^{6}$ \\
\hline \multicolumn{2}{|c|}{ For Adsorption of Levulinic Acid } \\
\hline Equation of $\mathrm{K}_{\mathrm{LA}}$ & $\mathrm{K}_{\mathrm{LA}}=4.59 * 10^{-4 *} \mathrm{e}^{\left(45347.88 / \mathrm{R}^{*} \mathrm{~T}\right)}$ \\
\hline Enthalpy of adsorption $\Delta H_{L A}^{0}(\mathrm{~kJ} / \mathrm{mol})$ & -45.347 \\
\hline $\mathrm{A}_{0 \mathrm{LA}}(\mathrm{lit} / \mathrm{mol})$ & $4.59 * 10^{-4}$ \\
\hline \multicolumn{2}{|c|}{ For Adsorption of Hydrogen } \\
\hline Equation of $\mathrm{K}_{\mathrm{H} 2}$ & $\mathrm{~K}_{\mathrm{H} 2}=4.93 * 10^{2} * \mathrm{e}^{(-7715.143 / \mathrm{R} * \mathrm{~T})}$ \\
\hline Enthalpy of adsorption $\Delta H_{H_{2}}^{0}(\mathrm{~kJ} / \mathrm{mol})$ & 7.7154 \\
\hline $\mathrm{A}_{0 \mathrm{H} 2}(\mathrm{lit} / \mathrm{mol})$ & $4.93 * 10^{2}$ \\
\hline
\end{tabular}


6] Figure S1: Py-IR of catalysts, a) Bare MMT, b) 0.5\% Ru/MMT, c) 5\% Ni/MMT, d) Fresh $0.5 \% \mathrm{Ru}-5 \% \mathrm{Ni} / \mathrm{MMT}$, e) Used $0.5 \% \mathrm{Ru}-5 \% \mathrm{Ni} / \mathrm{MMT}$.

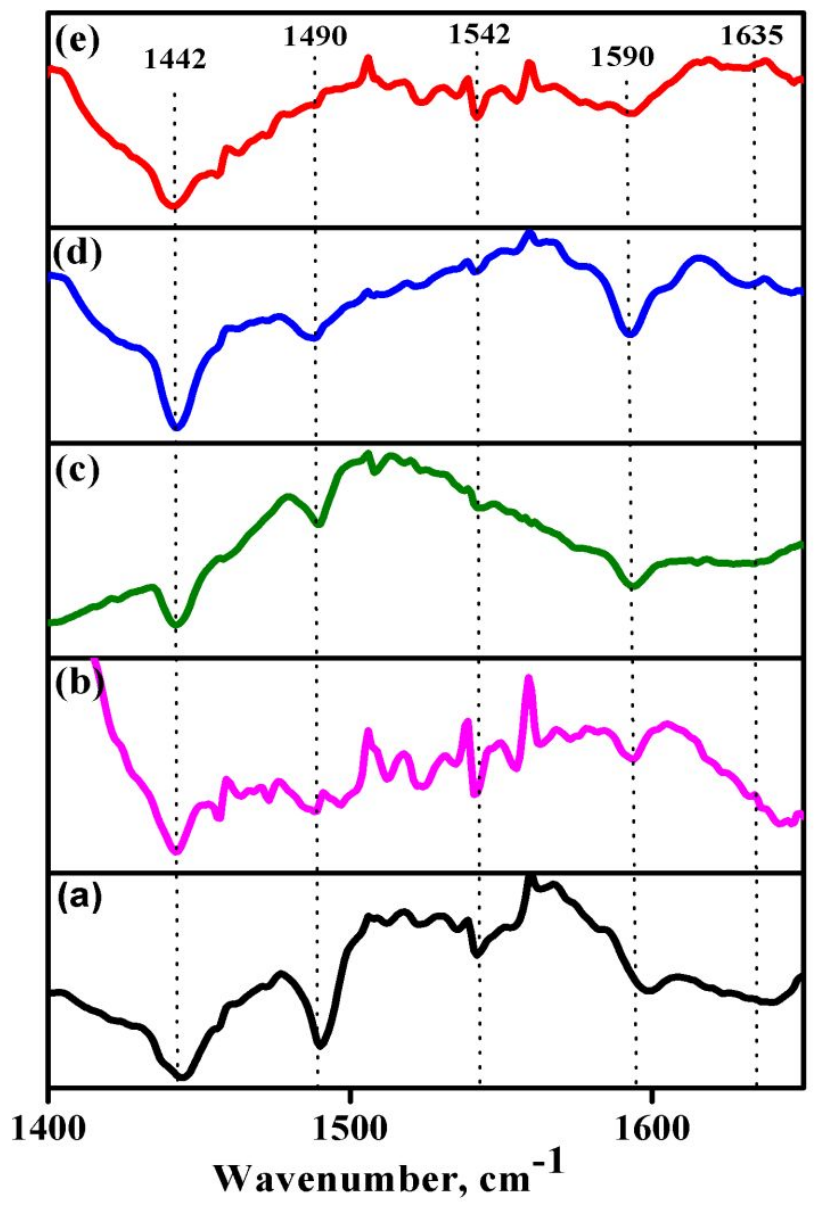


7] Figure S2: Concentration and selectivity Vs time profile of $0.5 \% \mathrm{Ru}-5 \% \mathrm{Ni} / \mathrm{MMT}$ catalyst.

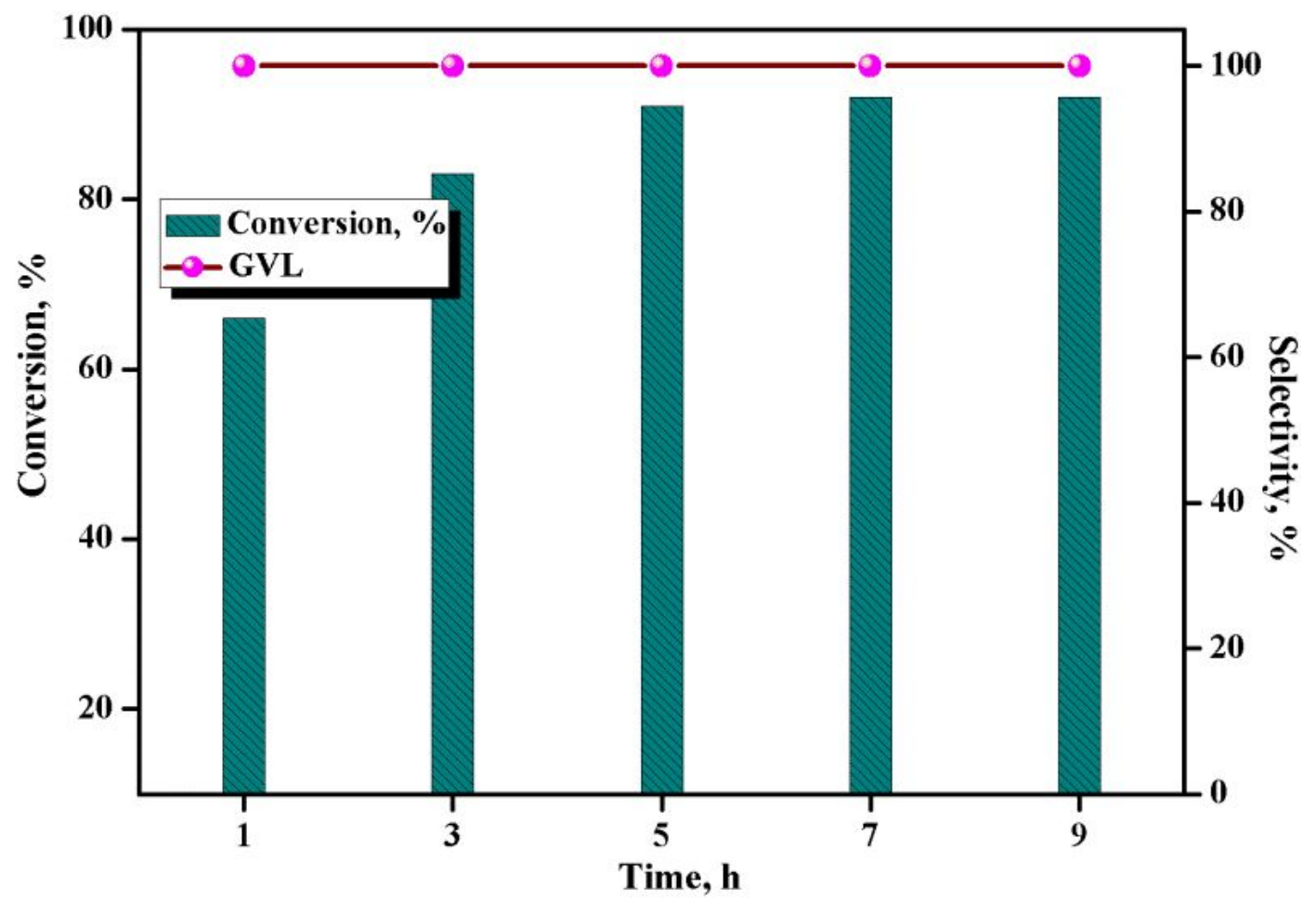

Reaction conditions: Levulinic Acid, 4g; Solvent, $95 \mathrm{~mL}$; catalyst, $0.25 \mathrm{~g}$; Temp, $220^{\circ} \mathrm{C} ; \mathrm{H}_{2}$ pressure, 250psi; agitation speed, 1000rpm. 
8] Figure S3: Effect of $0.5 \%$ Ru- 5\% Ni/MMT catalyst amount.

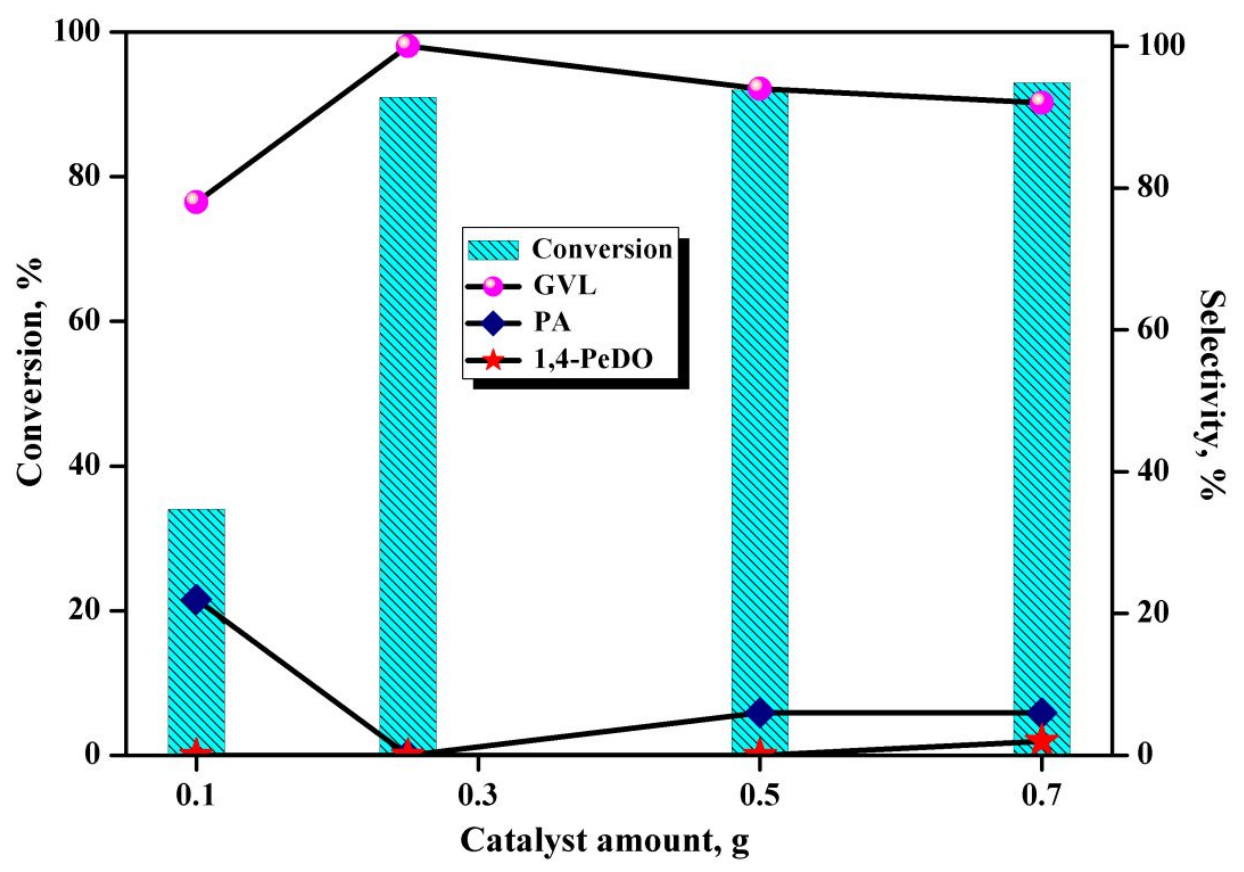

Reaction conditions: Levulinic Acid, 4g; Solvent, 95mL; Temp, $220^{\circ} \mathrm{C} ; \mathrm{H}_{2}$ pressure, 250psi; agitation speed, 1000rpm; reaction time, $5 \mathrm{~h}$. 
9] Figure S4: Graph of rate of reaction obtained from model-I (the first model considered) Vs rate of reaction obtained from experimental at temp $160^{\circ} \mathrm{C}$

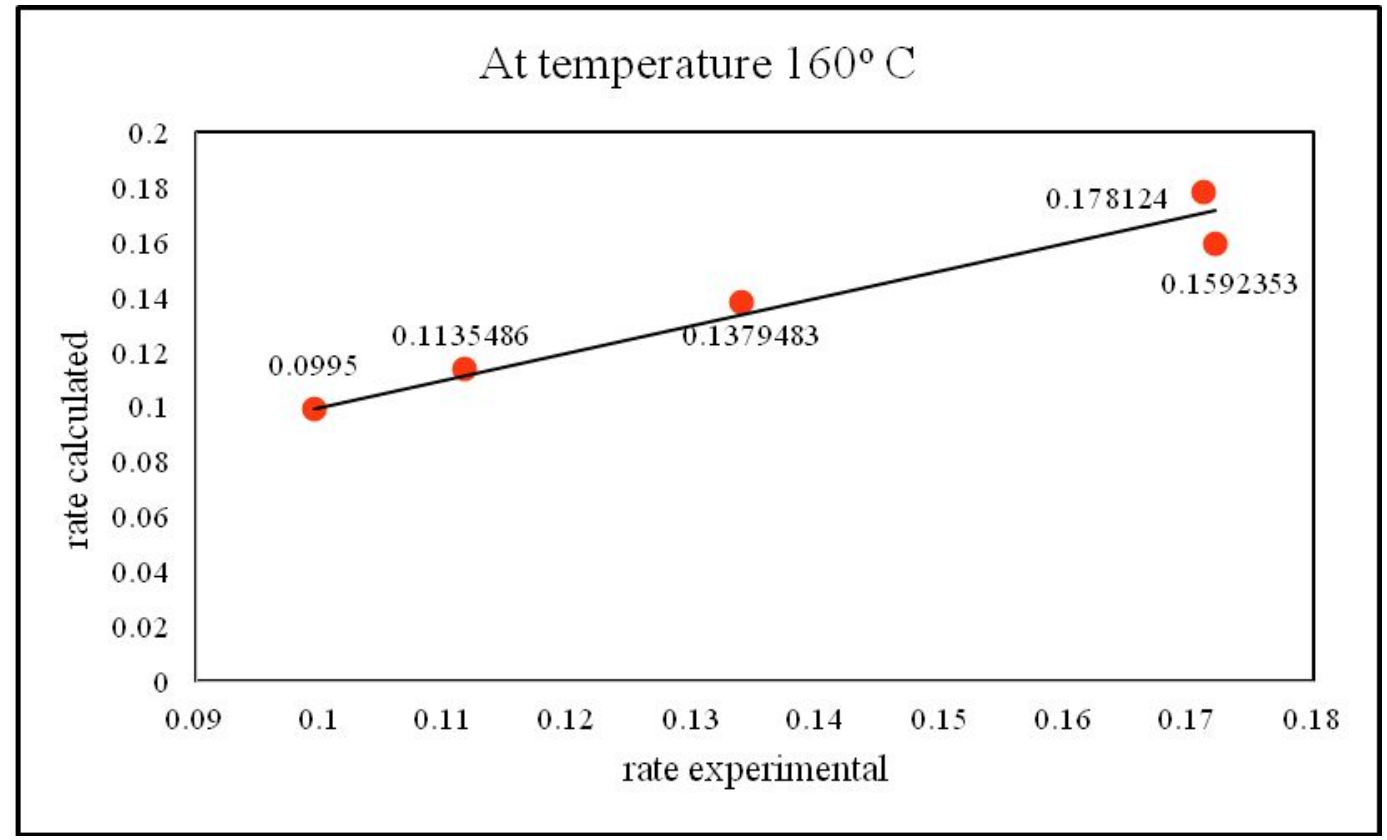

10] Figure S5: Graph of rate of reaction obtained from model-I (the first model considered) Vs rate of reaction obtained from experimental at temp $235^{\circ} \mathrm{C}$

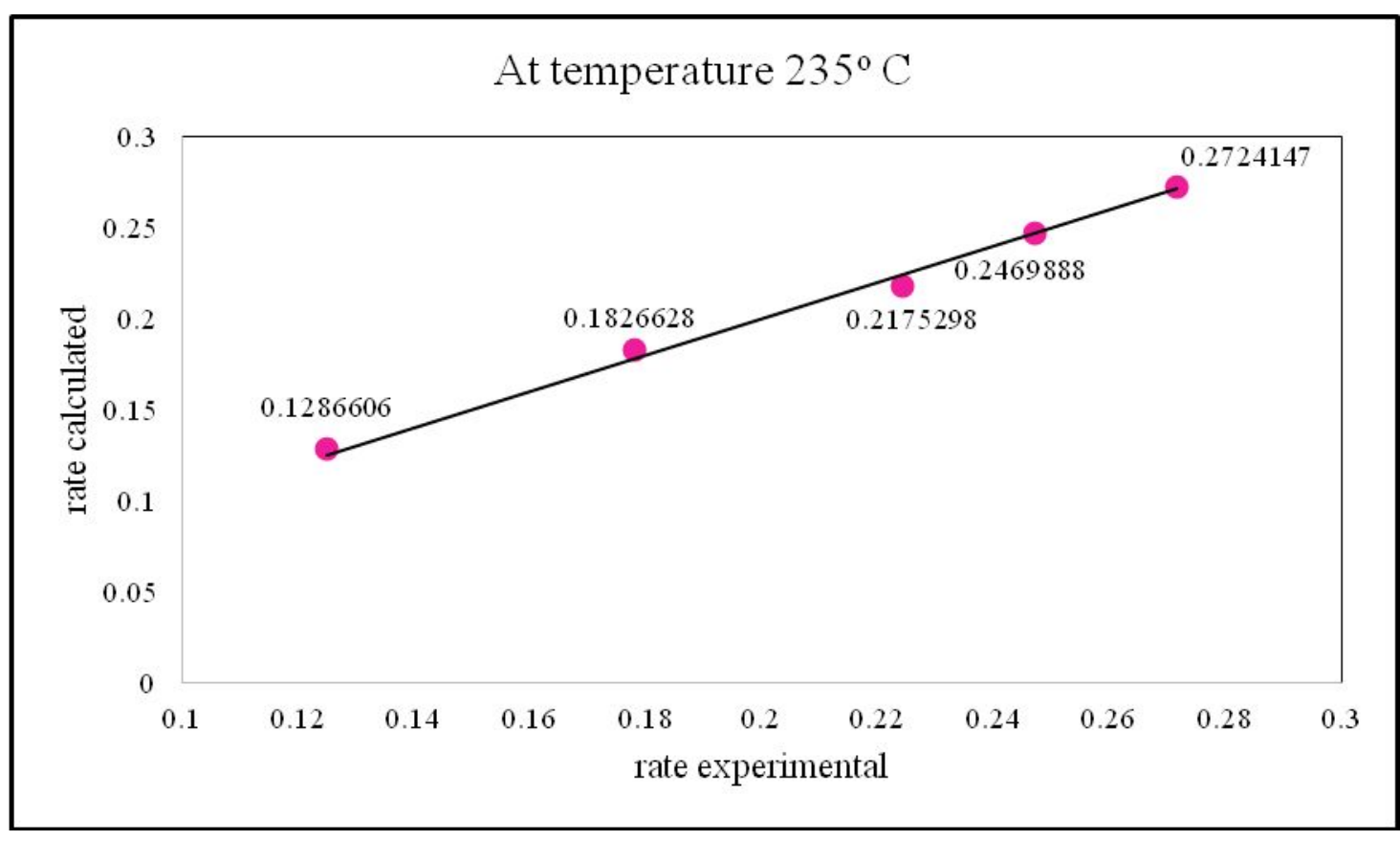


11] Figure S6: Arrhenius plot of rate constant $K_{L A}$ Vs $1 / T$ from Model I (the first model considered)

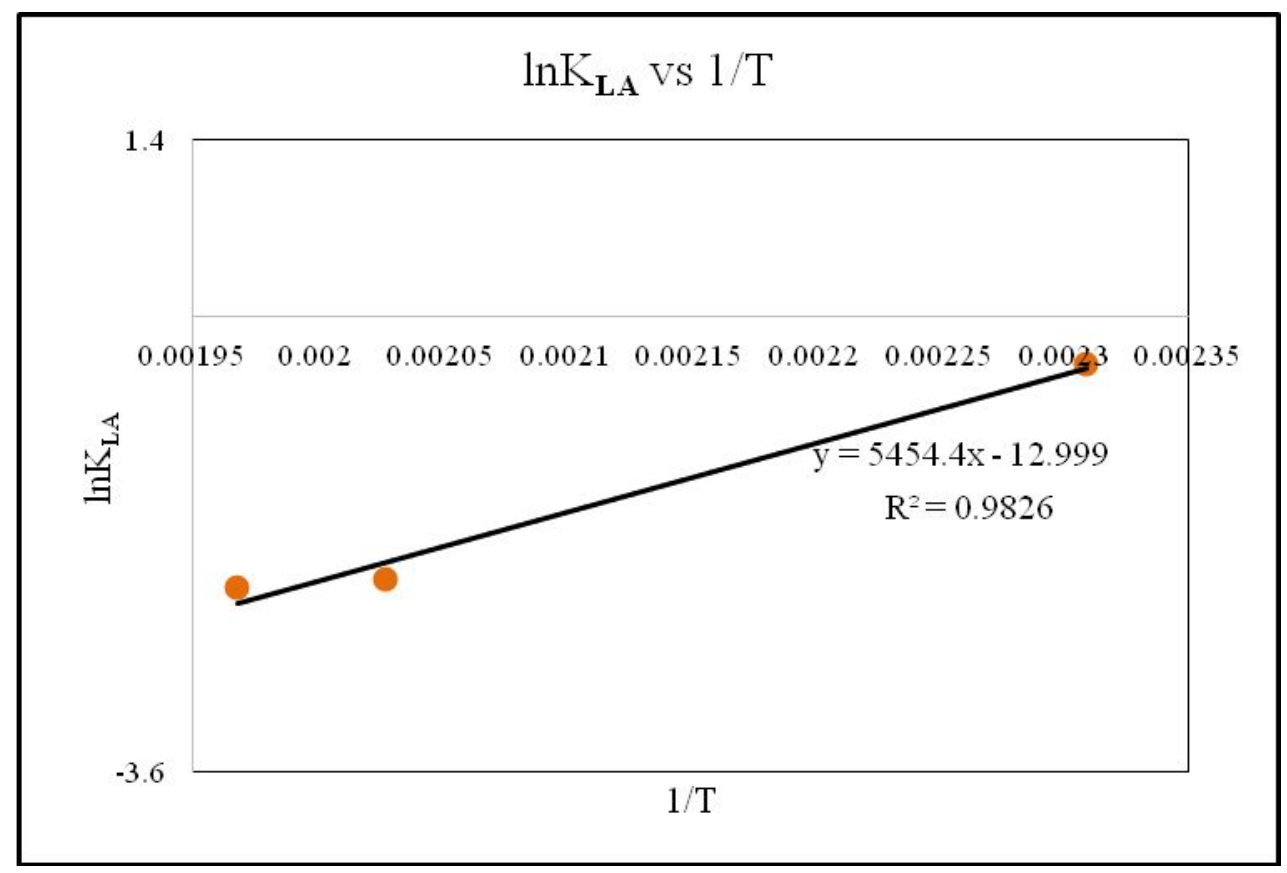

12] Figure $S 7$ : Arrhenius plot of rate constant $K_{H 2}$ Vs $1 / T$ from Model I(the first model considered)

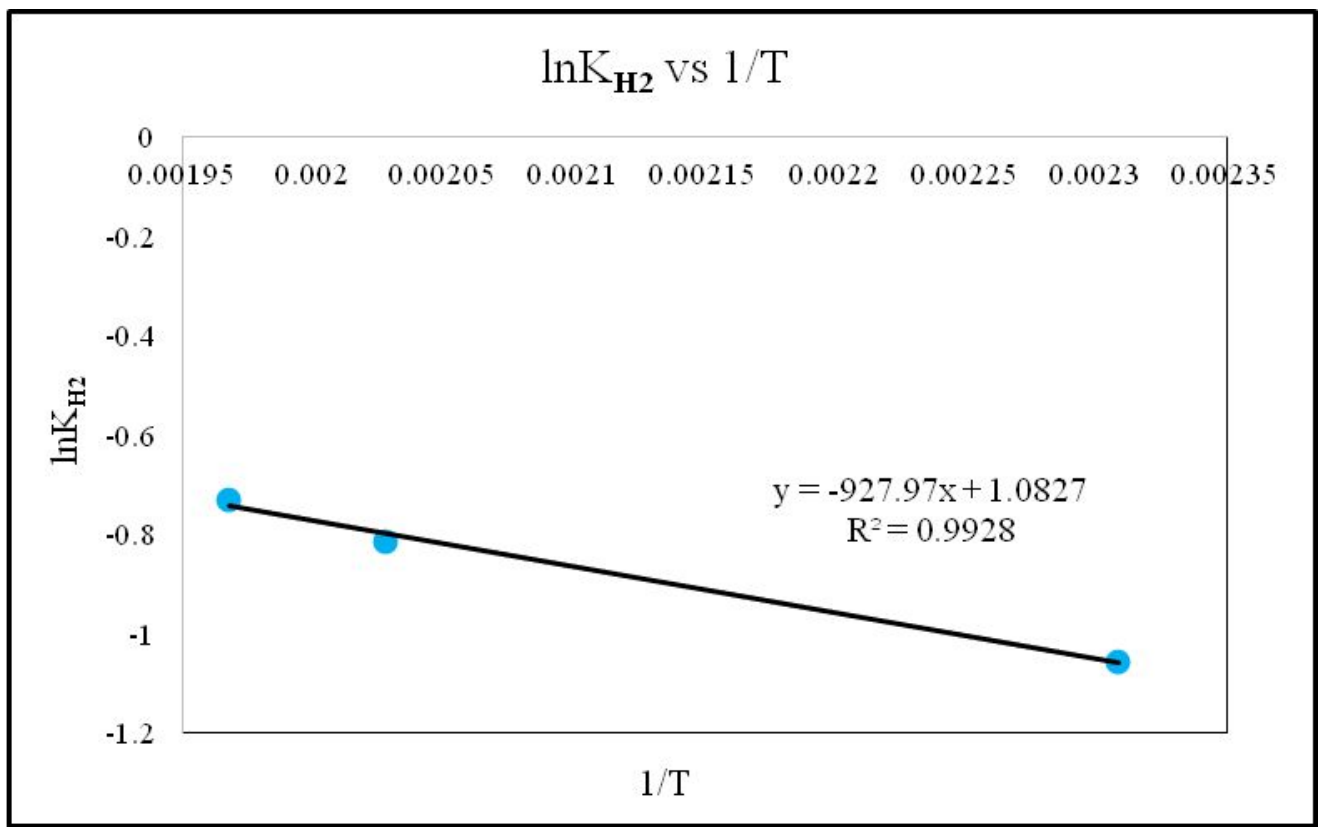


13] Figure S8: Arrhenius Plot for $\mathrm{k}_{3}$ obtained from Model II (the second model considered)

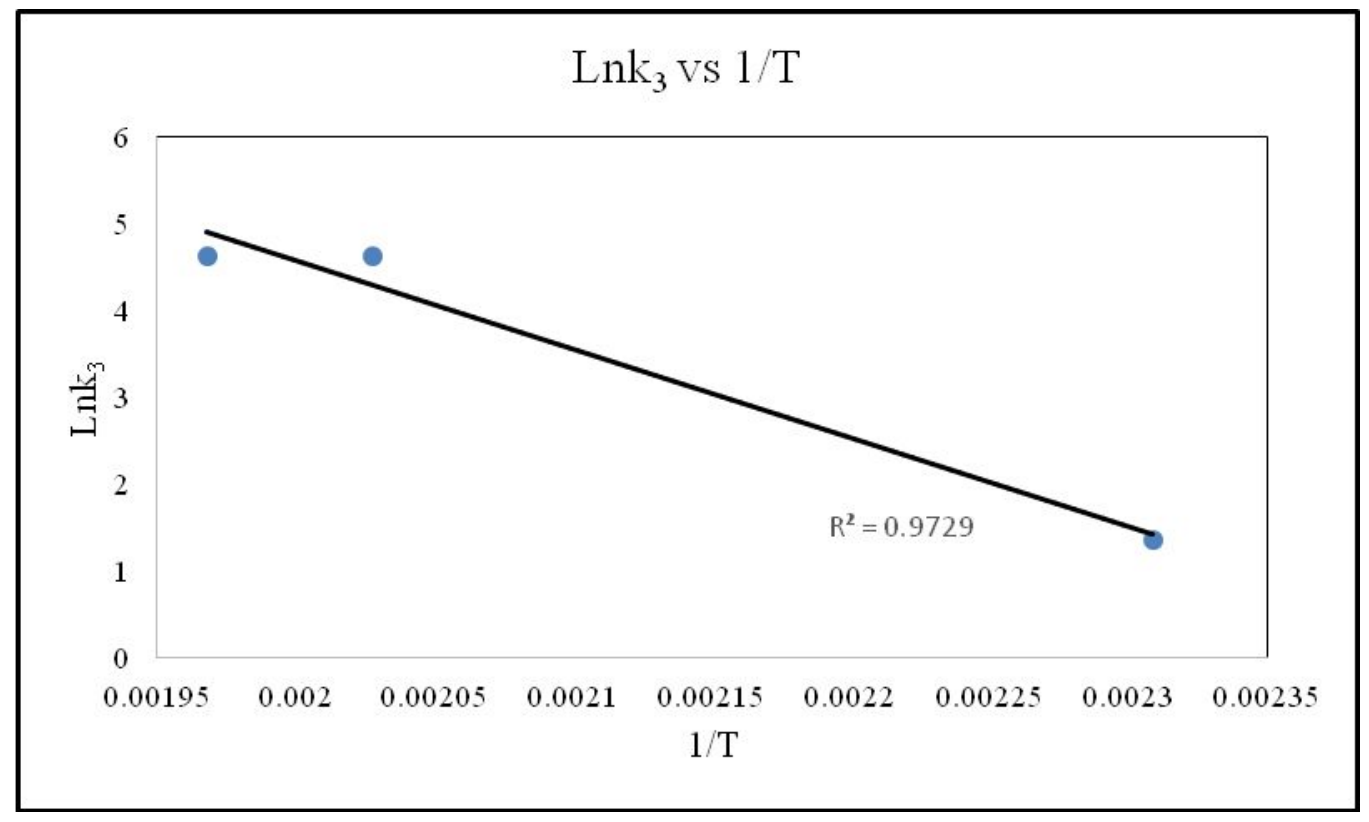

14] Figure S9: Arrhenius Plot for $\mathrm{K}_{\mathrm{LA}}$ obtained from Model II (the second model considered)

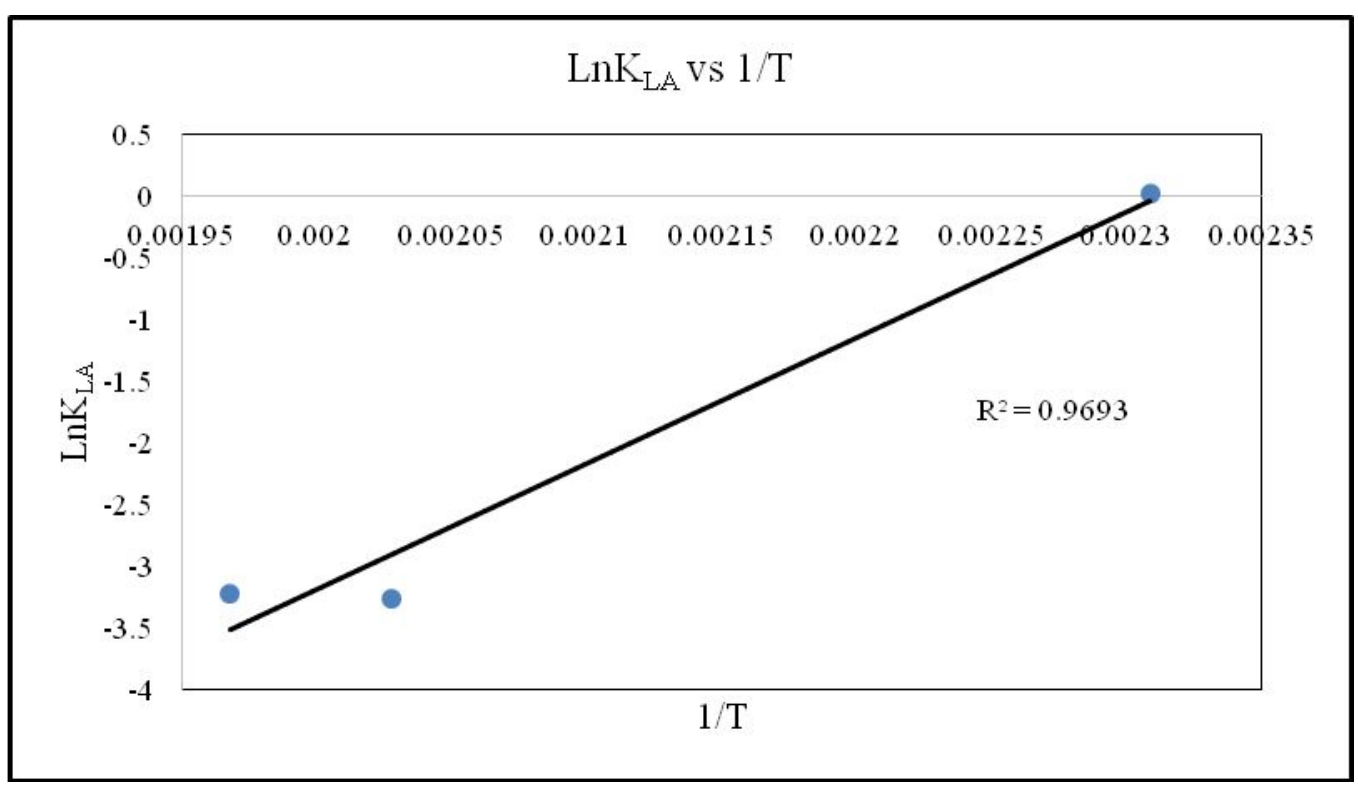


15] Figure S10: Arrhenius Plot for $\mathrm{K}_{\mathrm{H} 2}$ obtained from Model II (the second model considered)

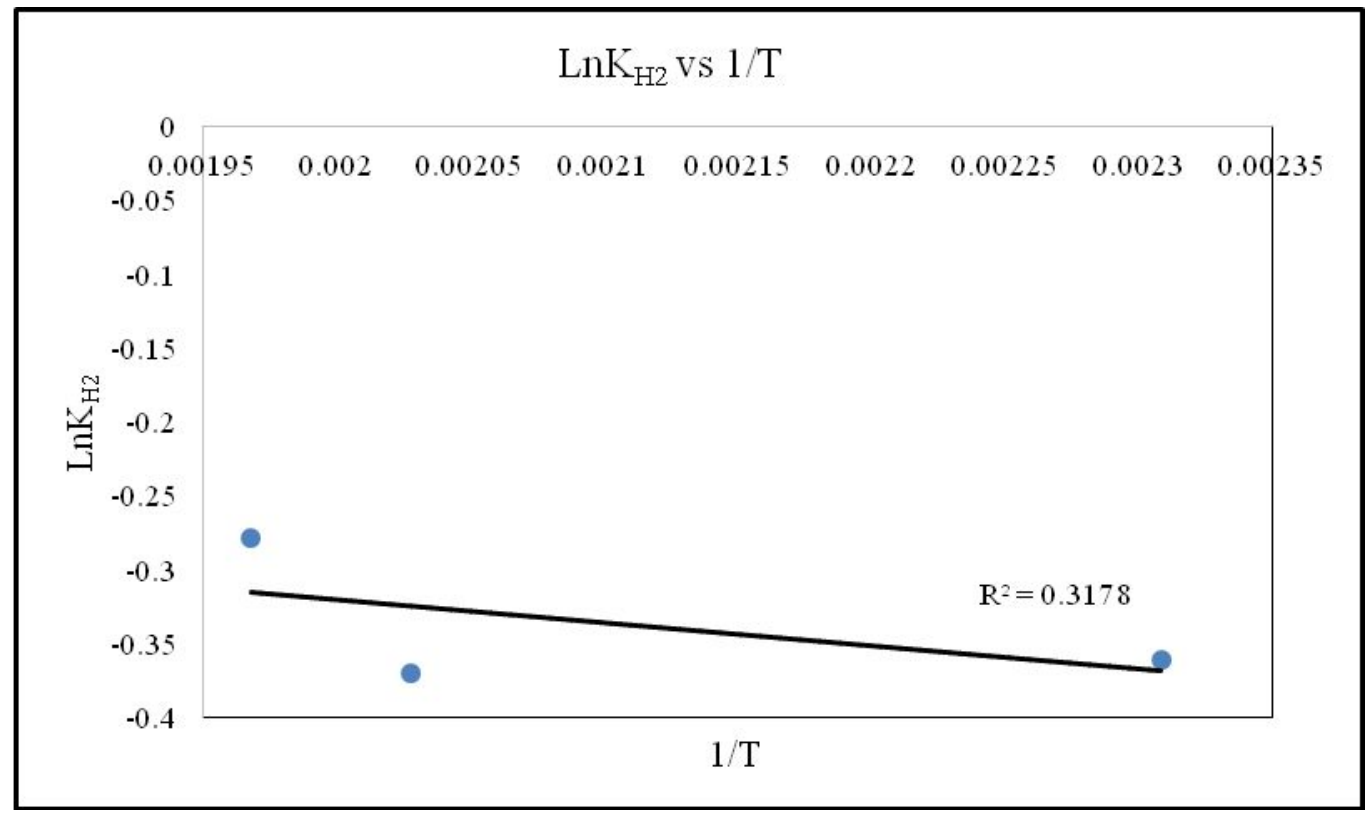


16] Figure S11: Detailed derivation of the LHHW models used

A. Model I : Considering Dissociative adsorption of $\mathrm{H}_{2}$ on catalyst surface, and assuming the chemical reaction on the catalyst surface as irreversible and rate limiting

The overall reaction is given by $L A+\mathrm{H}_{2} \leftrightarrow G V L+\mathrm{H}_{2} \mathrm{O}$

The individual steps according to LHHW Mechanism, assuming that the chemical reaction on the catalyst surface is irreversible, are given as:

$$
\begin{aligned}
& \mathrm{LA}+\theta_{v} \stackrel{\mathrm{k}_{1}, \mathrm{k}_{1}{ }^{*}}{\longrightarrow} \theta_{L A} \\
& H_{2}+2 \theta_{v} \stackrel{k_{2^{v}} \mathrm{k}_{2}^{x}}{\longrightarrow} 2 \theta_{H} \\
& 2 \theta_{H}+\theta_{L A} \stackrel{k_{3}}{\longrightarrow} \theta_{G V L}+\theta_{H_{2} O}+\theta_{v} \\
& \theta_{G V L} \stackrel{k_{4^{x}} \mathrm{k}_{4}{ }^{x}}{\longleftrightarrow} \theta_{V}+G V L \\
& \theta_{\mathrm{H}_{2} \mathrm{O}} \stackrel{k_{5}, \mathrm{k}_{5}{ }^{x}}{\longleftrightarrow} \theta_{V}+\mathrm{H}_{2} \mathrm{O}
\end{aligned}
$$

Thus the rate equations for all the individual steps of the reaction can be written as:

$$
\begin{aligned}
& -r_{1}=k_{1} L A \theta_{v}-k_{1}^{\prime} \theta_{L A} \\
& -r_{2}=k_{2} H_{2} \theta_{v}^{2}-k_{1}^{\prime} \theta_{H}^{2}
\end{aligned}
$$

$-r_{3}=k_{3} \theta_{L A} \theta_{H}^{2}$

$-r_{4}=k_{4} \theta_{G V L}-k_{4}^{\prime} \theta_{v} G V L$

$-r_{5}=k_{5} \theta_{H_{2} O}-k_{5}^{l} \theta_{v} H_{2} O$

The total concentration of active sites on the catalyst surface can be written as:

$$
\theta_{t}=1=\theta_{v}+\theta_{L A}+\theta_{H}+\theta_{G V L}+\theta_{H_{2} o}
$$

As we have assumed the surface chemical reaction step (c) to be Rate Determining we get,

$$
\frac{-r_{1}}{k_{1}}=0 \text { Thus, we get: } \theta_{L A}=\frac{k_{1}}{k_{1}^{\prime}} \theta_{v} L A=K_{L A} L A \theta_{v}
$$




$$
\begin{aligned}
& \frac{-r_{2}}{k_{2}}=0 \text { Thus, we get: } \theta_{H}=\sqrt{\frac{k_{2}}{k_{2}^{\prime}} \theta_{v}^{2} H_{2}}=\sqrt{K_{H_{2}} \theta_{v}^{2} H_{2}} \\
& \frac{-r_{4}}{k_{4}}=0 \text { Thus, we get: } \theta_{G V L}=\frac{k_{4}^{\prime}}{k_{4}} \theta_{v} G V L=K_{G V L}{ }^{\prime} \theta_{v} G V L \\
& \frac{-r_{5}}{k_{5}}=0 \text { Thus, we get: } \theta_{H_{2} O}=\frac{k_{5}^{\prime}}{k_{5}} \theta_{v} H_{2} O=K_{H_{2}} O^{\prime} \theta_{v} H_{2} O
\end{aligned}
$$

Substituting these values in equation (i) we get the value of $\theta_{v}$ as:

$$
\theta_{v}=\frac{\theta_{t}}{\left(1+K_{L A} L A+\sqrt{K_{H_{2}} H_{2}}+K_{G V L}{ }^{l} G V L+K_{H_{2} O}{ }^{\prime} H_{2} O\right)}
$$

Substituting the value of $\theta_{L A}, \theta_{H}$ and $\theta_{v}$ in $-r_{3}$ we get,

$$
-r_{3}=\frac{k_{3} K_{L A} L A K_{H_{2}} H_{2}}{\left(1+K_{L A} L A+\sqrt{K_{H_{2}} H_{2}}+K_{G V L}{ }^{\prime} G V L+K_{H_{2}} o^{\prime} H_{2} O\right)^{3}}
$$

Now, assuming initial rate method we get,

$$
-r_{3}=\frac{k_{3} K_{L A} L A_{0} K_{H_{2}} H_{2_{0}}}{\left(1+K_{L A} L A_{0}+\sqrt{K_{H_{2} H_{20}}}\right)^{3}} \quad \ldots . \text { Model I }
$$

B. Model II : Considering Non-Dissociative adsorption of $\mathrm{H}_{2}$ on catalyst surface and assuming the chemical reaction on the catalyst surface as irreversible and rate limiting

The overall reaction is given by $L A+\mathrm{H}_{2} \leftrightarrow \mathrm{GVL}+\mathrm{H}_{2} \mathrm{O}$

The individual steps according to LHHW Mechanism, assuming that the chemical reaction on the catalyst surface is irreversible, are given as:

$$
\begin{aligned}
& \mathrm{LA}+\theta_{v} \stackrel{k_{1}, \mathrm{k}_{1}{ }^{2}}{\longrightarrow} \theta_{L A} \\
& H_{2}+\theta_{v} \stackrel{k_{2}, \mathrm{k}_{2}{ }^{x}}{\longrightarrow} \theta_{H_{2}} \\
& \theta_{H_{2}}+\stackrel{\theta_{L A}}{\stackrel{k_{3}}{\longrightarrow}} \theta_{G V L}+\theta_{H_{2} O} \\
& \theta_{G V L} \stackrel{k_{4}, k_{4}{ }^{x}}{\longrightarrow} \theta_{V}+G V L
\end{aligned}
$$




$$
\theta_{\mathrm{H}_{2} \mathrm{O}} \stackrel{k_{5^{\prime}} \mathrm{k}_{5}{ }^{x}}{\longrightarrow} \theta_{V}+\mathrm{H}_{2} \mathrm{O}
$$

Thus the rate equations for all the individual steps of the reaction can be written as:

$$
\begin{aligned}
& -r_{1}=k_{1} L A \theta_{v}-k_{1}^{\prime} \theta_{L A} \\
& -r_{2}=k_{2} H_{2} \theta_{V}-k_{1}^{\prime} \theta_{H_{2}} \\
& -r_{3}=k_{3} \theta_{L A} \theta_{H_{2}} \\
& -r_{4}=k_{4} \theta_{G V L}-k_{4}^{\prime} \theta_{v} G V L \\
& -r_{5}=k_{5} \theta_{H_{2} O}-k_{5}^{\prime} \theta_{v} H_{2} O
\end{aligned}
$$

The total concentration of active sites on the catalyst surface can be written as:

$$
\theta_{t}=1=\theta_{v}+\theta_{L A}+\theta_{H_{2}}+\theta_{G V L}+\theta_{H_{2} O}
$$

As we have assumed the surface chemical reaction step (h) to be Rate Determining we get,

$$
\begin{gathered}
\frac{-r_{1}}{k_{1}}=0 \text { Thus, we get: } \theta_{L A}=\frac{k_{1}}{k_{1}^{\prime}} \theta_{v} L A=K_{L A} L A \theta_{v} \\
\frac{-r_{2}}{k_{2}}=0 \text { Thus, we get: } \theta_{H_{2}}=\frac{k_{2}}{k_{2}^{\prime}} \theta_{v} H_{2}=K_{H_{2}} \theta_{v} H_{2} \\
\frac{-r_{4}}{k_{4}}=0 \text { Thus, we get: } \theta_{G V L}=\frac{k_{4}^{\prime}}{k_{4}} \theta_{v} G V L=K_{G V L}{ }^{\prime} \theta_{v} G V L \\
\frac{-r_{5}}{k_{5}}=0 \text { Thus, we get: } \theta_{H_{2} O}=\frac{k_{5}^{\prime}}{k_{5}} \theta_{v} H_{2} O=K_{H_{2}} O^{\prime} \theta_{v} H_{2} O
\end{gathered}
$$

Substituting these values in equation (ii) we get the value of $\theta_{v}$ as:

$$
\theta_{v}=\frac{\theta_{t}}{\left(1+K_{L A} L A+K_{H_{2}} H_{2}+K_{G V L}{ }^{\prime} G V L+K_{H_{2} O}{ }^{\prime} H_{2} O\right)}
$$

Substituting the value of $\theta_{L A}, \theta_{H_{2}}$ and $\theta_{v}$ in $-r_{3}$ we get,

$-r_{3}=\frac{k_{3} K_{L A} L A K_{H_{2}} H_{2}}{\left(1+K_{L A} L A+K_{H_{2}} H_{2}+K_{G V L}{ }^{G} G V L+K_{H_{2}} o^{l} H_{2} O\right)^{2}}$

Now, assuming initial rate of reaction we get, 


$$
-r_{3}=\frac{k_{3} K_{L A} L A_{0} K_{H_{2}} H_{2_{0}}}{\left(1+K_{L A} L A_{0}+K_{H_{2}} H_{2_{0}}\right)^{2}}
$$

17] Figure S12: Report of POLYMATH 6.1 for model I(the first model considered) Model I: Dissociative adsorption of $\mathrm{H}_{2}$, Chemical Reaction as Rate Limiting Step $\left(220^{\circ} \mathrm{C}\right)$ 
POLYMATH Report

Nonlinear Regression (L-M)

Model: $\mathrm{ra}=\left(\mathrm{k} 3^{*}\left(\left(\mathrm{KLA} * \mathrm{CLA} 0^{\wedge} 1.0\right)^{*}\left(\mathrm{KH} 2^{*} \mathrm{CH} 20^{\wedge} 1\right)\right)\right) /\left(1+\left(\mathrm{KLA}^{*}\left(\mathrm{CLA} 0^{\wedge}(1)\right)\right)+\left(\mathrm{KH}^{*}{ }^{*} \mathrm{CH} 20\right)^{\wedge} 0.5\right)^{\wedge}(3)$

\begin{tabular}{|l|l|l|l|}
\hline Variable & Initial guess & Value & $\mathbf{9 5 \%}$ confidence \\
\hline K3 & 0.9 & 91.89999 & 0.1970326 \\
\hline KLA & 1. & 0.1247673 & 0.0002923 \\
\hline KH2 & 1. & 0.4428263 & 0.0016655 \\
\hline
\end{tabular}

Nonlinear regression settings

Max \# iterations $=300$

Precision

\begin{tabular}{|l|l|}
\hline$R^{\wedge} 2$ & 0.9922382 \\
\hline$R^{\wedge}$ 2adj & 0.9844764 \\
\hline Rmsd & 0.0019955 \\
\hline Variance & $4.978 E-05$ \\
\hline
\end{tabular}

General

\begin{tabular}{|l|l|}
\hline Sample size & 5 \\
\hline Model vars & 3 \\
\hline Indep vars & 2 \\
\hline Iterations & 112 \\
\hline
\end{tabular}

Source data points and calculated data points

\begin{tabular}{|l|l|l|l|l|l|}
\hline & CLA0 & CH20 & ra & ra calc & Delta ra \\
\hline 1 & 0.344500904 & 0.252099315 & 0.167924528 & 0.1688546 & -0.0009301 \\
\hline 2 & 0.344500904 & 0.33613242 & 0.2009048 & 0.2015785 & -0.0006737 \\
\hline 3 & 0.344500904 & 0.420165525 & 0.234210526 & 0.2293383 & 0.0048722 \\
\hline 4 & 0.344500904 & 0.50419863 & 0.254285714 & 0.2533811 & 0.0009046 \\
\hline 5 & 0.172250452 & 0.420165525 & 0.11125 & 0.1198336 & -0.0085836 \\
\hline
\end{tabular}

Model I: Dissociative adsorption of $\mathrm{H}_{2}$, Chemical Reaction as rate limiting Step $\left(160^{\circ} \mathrm{C}\right)$ 
POLYMATH Report

Nonlinear Regression (L-M)

Model: $\mathrm{ra}=\left(\mathrm{k} 3 *\left(\left(\mathrm{KLA} * \mathrm{CLA} 0^{\wedge} 1.0\right) *\left(\mathrm{KH} 2 * \mathrm{CH} 20^{\wedge} 1\right)\right)\right) /\left(1+\left(\mathrm{KLA}^{*}\left(\mathrm{CLA} 0^{\wedge}(1)\right)\right)+(\mathrm{KH} 2 * \mathrm{CH} 20)^{\wedge} 0.5\right) \wedge(3)$

\begin{tabular}{|l|l|l|l|}
\hline Variable & Initial guess & Value & $\mathbf{9 5 \%}$ confidence \\
\hline k3 & 1. & 18.05514 & 14.95761 \\
\hline KLA & 1. & 0.6830999 & 0.2815356 \\
\hline KH2 & 5. & 0.3474951 & 0.150819 \\
\hline
\end{tabular}

Nonlinear regression settings

Max \# iterations $=300$

Precision

\begin{tabular}{|l|l|}
\hline$R^{\wedge} 2$ & 0.9478842 \\
\hline
\end{tabular}

$R^{\wedge}$ 2adj 0.8957685

\begin{tabular}{|l|l|}
\hline Rmsd & 0.0030474 \\
\hline
\end{tabular}

Variance 0.0001161

\section{General}

Sample size 5

Model vars 3

Indep vars 2

Iterations 69

Source data points and calculated data points

\begin{tabular}{|l|l|l|l|l|l|}
\hline & CLA0 & CH20 & ra & ra calc & Delta ra \\
\hline 1 & 0.344500904 & 0.287020148 & 0.1115217 & 0.1135486 & -0.0020269 \\
\hline 2 & 0.344500904 & 0.382693531 & 0.1338824 & 0.1379483 & -0.0040659 \\
\hline 3 & 0.344500904 & 0.574040296 & 0.171153846 & 0.178124 & -0.0069702 \\
\hline 4 & 0.344500904 & 0.478366913 & 0.172 & 0.1592353 & 0.0127647 \\
\hline 5 & 0.172250452 & 0.4783669 & 0.0995 & 0.0995 & $-1.641 \mathrm{E}-12$ \\
\hline
\end{tabular}

Model I: Dissociative adsorption of $\mathrm{H}_{2}$, Chemical Reaction as rate limiting Step $\left(235^{\circ} \mathrm{C}\right)$ 
POLYMATH Report

Nonlinear Regression (L-M)

Model: $\mathrm{ra}=\left(\mathrm{k}^{*} *\left(\left(\mathrm{KLA} * \mathrm{CLA} 0^{\wedge} 1.0\right) *\left(\mathrm{KH}^{*} \mathrm{CH}^{20} 0^{\wedge} 1\right)\right)\right) /\left(1+\left(\mathrm{KLA}^{*}\left(\mathrm{CLA} 0^{\wedge}(1)\right)\right)+(\mathrm{KH} 2 * \mathrm{CH} 20)^{\wedge} 0.5\right) \wedge(3)$

\begin{tabular}{|l|l|l|l|}
\hline Variable & Initial guess & Value & $\mathbf{9 5 \%}$ confidence \\
\hline K3 & 1. & 101.9975 & 4.990008 \\
\hline KLA & 1. & 0.1168427 & 0.0062069 \\
\hline KH2 & 1. & 0.4815509 & 0.0419487 \\
\hline
\end{tabular}

Nonlinear regression settings

Max \# iterations $=300$

\section{Precision}

\begin{tabular}{|l|l|}
\hline$R^{\wedge} 2$ & 0.9941254 \\
\hline$R^{\wedge}$ 2adj & 0.9882507 \\
\hline Rmsd & 0.0017909 \\
\hline Variance & $4.009 E-05$ \\
\hline
\end{tabular}

General

\begin{tabular}{|l|l|}
\hline Sample size & 5 \\
\hline Model vars & 3 \\
\hline Indep vars & 2 \\
\hline Iterations & 130 \\
\hline
\end{tabular}

Source data points and calculated data points

\begin{tabular}{|l|l|l|l|l|l|}
\hline & CLA0 & CH20 & ra & ra calc & Delta ra \\
\hline 1 & 0.344500904 & 0.244657635 & 0.178 & 0.1826628 & -0.0046628 \\
\hline 2 & 0.344500904 & 0.32621018 & 0.2242051 & 0.2175298 & 0.0066753 \\
\hline 3 & 0.344500904 & 0.407762725 & 0.2472254 & 0.2469888 & 0.0002366 \\
\hline 4 & 0.344500904 & 0.48931527 & 0.2717647 & 0.2724147 & -0.00065 \\
\hline 5 & 0.172250452 & 0.407762725 & 0.125 & 0.1286606 & -0.0036606 \\
\hline
\end{tabular}

18] Figure S13: Report of POLYMATH 6.1 for model II (the second model considered) 
Model II: Non-Dissociative adsorption of $\mathrm{H}_{2}$, Chemical Reaction as rate limiting Step $\left(220^{\circ} \mathrm{C}\right)$

POLYMATH Report

Nonlinear Regression (L-M)

Model: $\quad r a=\left(k 3^{*}\left(\left(\mathrm{KLA}^{*} \mathrm{CLA0} 0^{\wedge} 1.0\right)^{*}\left(\mathrm{KH} 2^{*} \mathrm{CH} 20^{\wedge} 1\right)\right)\right) /\left(1+\left(\mathrm{KLA}^{*}\left(\mathrm{CLA} 0^{\wedge}(1)\right)\right)+\left(\mathrm{KH}^{*}\left(\mathrm{CH} 20^{\wedge}(1)\right)\right)\right)^{\wedge}(2)$

\begin{tabular}{|l|l|l|l|}
\hline Variable & Initial guess & Value & $\mathbf{9 5} \%$ confidence \\
\hline K3 & 0.9 & 101.9993 & 3.601274 \\
\hline KLA & 1. & 0.0384834 & 0.0017131 \\
\hline KH2 & 1. & 0.690822 & 0.0471498 \\
\hline
\end{tabular}

Nonlinear regression settings

Max \# iterations $=300$

Precision

\begin{tabular}{|l|l|}
\hline$R^{\wedge} 2$ & 0.9710439 \\
\hline$R^{\wedge}$ 2adj & 0.9420878 \\
\hline Rmsd & 0.003731 \\
\hline Variance & 0.000174 \\
\hline
\end{tabular}

General

\begin{tabular}{|l|l|}
\hline Sample size & 5 \\
\hline Model vars & 3 \\
\hline Indep vars & 2 \\
\hline Iterations & 141 \\
\hline
\end{tabular}

Source data points and calculated data points

\begin{tabular}{|l|l|l|l|l|l|}
\hline & CLA0 & CH20 & ra & ra calc & Delta ra \\
\hline 1 & 0.344500904 & 0.252099315 & 0.167924528 & 0.1670311 & 0.0008935 \\
\hline 2 & 0.344500904 & 0.33613242 & 0.2009048 & 0.2024206 & -0.0015158 \\
\hline 3 & 0.344500904 & 0.420165525 & 0.234210526 & 0.2309801 & 0.0032304 \\
\hline 4 & 0.344500904 & 0.50419863 & 0.254285714 & 0.254034 & 0.0002517 \\
\hline 5 & 0.172250452 & 0.420165525 & 0.11125 & 0.1168041 & -0.0055541 \\
\hline
\end{tabular}

Model II: Non-Dissociative adsorption of $\mathrm{H}_{2}$, Chemical Reaction as rate limiting Step $\left(160^{\circ} \mathrm{C}\right)$ 
POLYMATH Report

Nonlinear Regression (L-M)

Model: $r a=\left(k 3 *\left(\left(K L A^{*} C L A 0^{\wedge} 1.0\right) *\left(\mathrm{KH}^{*} \mathrm{CH}^{2} 0^{\wedge} 1\right)\right)\right) /\left(1+\left(\mathrm{KLA}^{*}(\mathrm{CLA} 0 \wedge(1))\right)+\left(\mathrm{KH}^{*}\left(\mathrm{CH} 20^{\wedge}(1)\right)\right)\right)^{\wedge}(2)$

\begin{tabular}{|l|l|l|l|}
\hline Variable & Initial guess & Value & $\mathbf{9 5 \%}$ confidence \\
\hline K3 & 1. & 3.882916 & 10.82633 \\
\hline KLA & 1. & 1.014883 & 2.875596 \\
\hline KH2 & 1. & 0.6970957 & 1.694802 \\
\hline
\end{tabular}

Nonlinear regression settings

Max \# iterations $=300$

\section{Precision}

\begin{tabular}{|l|l|}
\hline$R^{\wedge} 2$ & 0.9522053 \\
\hline$R^{\wedge}$ 2adj & 0.9044105 \\
\hline Rmsd & 0.0029183 \\
\hline Variance & 0.0001065 \\
\hline
\end{tabular}

\section{General}

\begin{tabular}{|l|l|}
\hline Sample size & 5 \\
\hline Model vars & 3 \\
\hline Indep vars & 2 \\
\hline Iterations & 14 \\
\hline
\end{tabular}

Source data points and calculated data points

\begin{tabular}{|l|l|l|l|l|l|}
\hline & CLAO & CH20 & ra & ra calc & Delta ra \\
\hline 1 & 0.344500904 & 0.287020148 & 0.1115217 & 0.1131017 & -0.00158 \\
\hline 2 & 0.344500904 & 0.382693531 & 0.1338824 & 0.1386146 & -0.0047322 \\
\hline 3 & 0.344500904 & 0.574040296 & 0.171153846 & 0.1774303 & -0.0062764 \\
\hline 4 & 0.344500904 & 0.478366913 & 0.172 & 0.1598087 & 0.0121913 \\
\hline 5 & 0.172250452 & 0.4783669 & 0.0995 & 0.0995 & $3.229 \mathrm{E}-09$ \\
\hline
\end{tabular}

Model II: Non-Dissociative adsorption of $\mathrm{H}_{2}$, Chemical Reaction as rate limiting Step $\left(235^{\circ} \mathrm{C}\right)$ 
POLYMATH Report

Nonlinear Regression (L-M)

Model: $\mathrm{ra}=\left(\mathrm{k} 3^{*}\left(\left(\mathrm{KLA}{ }^{*} \mathrm{CLA} 0^{\wedge} 1.0\right) *\left(\mathrm{KH} 2 * \mathrm{CH}^{2} 0^{\wedge} 1\right)\right)\right) /\left(1+\left(\mathrm{KLA}^{*}\left(\mathrm{CLA} 0^{\wedge}(1)\right)\right)+\left(\mathrm{KH} 2 *\left(\mathrm{CH} 20^{\wedge}(1)\right)\right)\right)^{\wedge}(2)$

\begin{tabular}{|l|l|l|l|}
\hline Variable & Initial guess & Value & $\mathbf{9 5 \%}$ confidence \\
\hline K3 & 1. & 101.9992 & 10.87501 \\
\hline KLA & 1. & 0.0400862 & 0.0043596 \\
\hline KH2 & 1. & 0.7568862 & 0.139183 \\
\hline
\end{tabular}

Nonlinear regression settings

Max \# iterations $=300$

Precision

\begin{tabular}{|l|l|}
\hline$R^{\wedge} 2$ & 0.9968216 \\
\hline$R^{\wedge} 2 a d j$ & 0.9936432 \\
\hline Rmsd & 0.0013173 \\
\hline Variance & $2.169 \mathrm{E}-05$ \\
\hline
\end{tabular}

General

\begin{tabular}{|l|l|}
\hline Sample size & 5 \\
\hline Model vars & 3 \\
\hline Indep vars & 2 \\
\hline Iterations & 267 \\
\hline
\end{tabular}

Source data points and calculated data points

\begin{tabular}{|l|l|l|l|l|l|}
\hline & CLA0 & CH20 & ra & ra calc & Delta ra \\
\hline 1 & 0.344500904 & 0.244657635 & 0.178 & 0.1814437 & -0.0034437 \\
\hline 2 & 0.344500904 & 0.32621018 & 0.2242051 & 0.2188151 & 0.00539 \\
\hline 3 & 0.344500904 & 0.407762725 & 0.2472254 & 0.2485813 & -0.0013559 \\
\hline 4 & 0.344500904 & 0.48931527 & 0.2717647 & 0.272286 & -0.0005213 \\
\hline 5 & 0.172250452 & 0.407762725 & 0.125 & 0.1255988 & -0.0005988 \\
\hline
\end{tabular}

